\title{
Toujours la même chanson : Les logiques mimétiques des radios musicales françaises
}

\author{
Philippe Mouricou ${ }^{1}$,
}

CREPA (Centre de Recherche en Management \& Organisation),

Université Paris Dauphine,

Place du Maréchal de Lattre de Tassigny

75775 Paris cedex 16

philippe.mouricou@dauphine.fr

Tél : + $33(0) 144054739$

Fax : + $33(0) 144054084$

Résumé :

Si l'imitation joue un rôle central dans les travaux sur la diffusion, la question des logiques mimétiques (raisons amenant les décideurs à reprendre des solutions adoptées ailleurs) y demeure subalterne. S'appuyant sur la théorie des conventions, les modèles de herding behavior, les théories de l'identité sociale et les théories néo-institutionnalistes, cet article propose une typologie des logiques mimétiques qui permet d'interpréter des données issues d'entretiens menés auprès de programmateurs radio. L'analyse montre que les trois grandes logiques, utilitariste, conventionnelle et référentielle, sont concomitantes.

Mots clés : Relations interorganisationnelles ; Imitation ; Logiques mimétiques ; Radio

\begin{abstract}
:
The literature on imitation is often grounded in a 'diffusion' perspective that focuses on effects and modes of imitation and gives little empirical attention to the reasons why managers imitate other firms. In this study of the French radio broadcasting industry, a typology of music programmers' mimetic rationales is proposed. Drawing on conventions, herding behavior, social identity and neoinstitutional theories, three distinct rationales associated with imitation are identified (utilitarian, conventional and referential). Our study shows that they work simultaneously.
\end{abstract}

Key words : Interorganizational relations ; Imitation ; Mimetic rationales ; Radio broadcasting industry

\footnotetext{
${ }^{1}$ L'auteur tient à remercier Bernard Forgues et deux évaluateurs anonymes de ce numéro pour leurs critiques constructives ainsi que Bernard de Montmorillon, Pierre Romelaer, et Jean-François Chanlat pour leurs remarques sur une version précédente de cet article.
} 
$X^{\text {ème }}$ Conférence Internationale de Management Stratégique, Annecy / Genève 13-16 Juin 2006

De l'imitation interorganisationnelle, les théories de la diffusion proposent aujourd'hui une image détaillée et étayée par de nombreux résultats empiriques. Le nombre grandissant de travaux s'inscrivant dans cette thématique ne saurait cependant occulter la stigmatisation dont a traditionnellement fait l'objet l'imitation en stratégie. A l'instar des auteurs de ce manuel de référence en management stratégique pour qui « une entreprise [qui] a la même stratégie que ses concurrents n'a pas de stratégie » (Johnson et al., $2005:$ 7), une abondante littérature considère l'innovation et la différenciation comme les seules alternatives permettant d'obtenir un avantage concurrentiel. Dès lors, comment interpréter l'attitude des dirigeants qui choisissent de s'engouffrer dans une voie qui serait vouée à l'échec? Quelles sont les logiques sous-jacentes à l'imitation (désormais, logiques mimétiques) ?

Plusieurs courants théoriques proposent des éléments de réponse à ces questions. Porteurs d'une conception calculatoire de la rationalité, les travaux traitant de l'innovation voient souvent en l'imitation une démarche stratégique visant à diminuer les coûts de recherche et développement ou à réduire la prise de risque associée à l'innovation (Levitt, 1966 ; Schnaars, 1994). A l'opposé, la théorie des conventions (Gomez, 1996), la littérature néoinstitutionnaliste (DiMaggio et Powell, 1983) et la théorie de l'autocatégorisation (Turner, 1999) intègrent des dimensions sociales et psycho-sociales mises de coté par les approches utilitaristes.

Ancré dans le paradigme interprétativiste, cet article vise à améliorer la compréhension de l'imitation interorganisationnelle en proposant une typologie des logiques mimétiques. Pour mener notre travail d'investigation empirique, nous avons retenu un champ d'étude atypique : les radios musicales françaises. Accusées d'être devenues de véritables «mee-too products, robotisés, désincarnés et markétés à outrance »(Blachas, $2004: 4)$, ces dernières auraient renoncé à toute différenciation. Vingt entretiens ont donc été menés auprès de directeurs des programmes et de responsables de la programmation musicale. Leur analyse révèle que les trois logiques mimétiques (conventionnelle, référentielle et utilitariste) agissent de façon concomitante dans l'industrie étudiée. Cette observation permet de poser le problème de l'incomplétude des approches théoriques actuelles.

Après quelques éléments de définition, nous évoquerons le cadre théorique de la recherche et notre typologie des logiques mimétiques. Nous reviendrons ensuite sur le secteur d'activité étudié et les choix méthodologiques retenus dans cette recherche. Suivront le détail des résultats et une discussion. 
$X^{\text {ème }}$ Conférence Internationale de Management Stratégique, Annecy / Genève 13-16 Juin 2006

\section{IMITATION INTERORGANISATIONNELLE : UNE DEFINITION}

Dans son sens le plus commun, l'imitation consiste en l'action de reproduire ou de chercher à reproduire (une apparence, un acte, un geste d'autrui). Imiter, c'est refaire ce que quelqu'un d'autre a déjà fait avant soi. Qu'elle soit immédiate ou différée, «l'imitation suppose que le comportement modèle soit décodé et interprété de façon suffisamment correcte pour que production et reproduction soient perçues comme semblables » (Baudonnière, 1997 : 43).

Comme l'explique Ellsworth (1926: 368) : «L'imitation doit clairement impliquer une similarité de comportement [...], imiter quelqu'un, c'est agir comme lui, il en est ainsi lorsque nous copions son style vestimentaire, que nous reproduisons ses mouvements, que nous essayons de penser à sa façon, d'avoir des sentiments ou des émotions qui ressemblent aux siens ». Le sociologue de mettre en garde ses lecteurs quant à l'utilisation inappropriée du terme : les mêmes causes, ou les mêmes mécanismes peuvent pousser les individus à adopter les mêmes comportements sans que l'on puisse parler d'imitation.

Appliquée au domaine des relations interorganisationnelles, l'imitation peut se définir par la séquence suivante (Haunschild, 1993) : (1) une organisation modèle adopte une pratique donnée à la date t ; (2) l'organisation imitante est exposée au modèle ; (3) l'organisation imitante adopte la pratique en $\mathrm{t}+\mathrm{x}$ ( $\mathrm{x}$ désignant un laps de temps positif).

Pour intégrer la remarque d'Ellsworth, il semble nécessaire d'ajouter à cette séquence une condition : l'existence d'un lien de causalité entre l'adoption de la pratique par l'organisation modèle, et son adoption par l'organisation imitante.

\section{CADRE THEORIQUE}

Les processus de diffusion des pratiques au sein de groupes d'organisations donnent lieu à un nombre croissant de recherches en sciences de gestion (pour un panorama complet, voir Strang et Soule [1998] et Greve [2005]). Adoptant le plus souvent une démarche quantitative, ces travaux cherchent à mettre en lumière l'existence de facteurs facilitant la diffusion (ex : adoption préalable de la pratique par une organisation performante chez Haveman [1993] ; appartenance à un même groupe chez Greve [1996]). Parce qu'ils renvoient souvent à des comportements mimétiques, ces travaux ont suscité un fort intérêt académique envers l'imitation interorganisationnelle, rompant ainsi avec la stigmatisation dont le phénomène a souvent fait l'objet. 
$X^{\text {ème }}$ Conférence Internationale de Management Stratégique, Annecy / Genève 13-16 Juin 2006

\subsection{LES TROIS FORMES D'IMITATION INTERORGANISATIONNELLE}

A partir de la littérature néo-institutionnaliste, des travaux sur la diffusion des pratiques et de la littérature sur l'apprentissage interorganisationnel, Haunschild et Miner (1997) construisent une typologie des formes d'imitation interorganisationnelle. Sont distinguées l'imitation basée sur la fréquence, l'imitation basée sur les résultats et l'imitation basée sur les caractéristiques du modèle. L'imitation basée sur la fréquence consiste pour une organisation à copier les pratiques les plus largement diffusées. L'imitation basée sur les résultats consiste pour une organisation à copier les pratiques qui semblent avoir connu de bons résultats par le passé. L'imitation basée sur les caractéristiques du modèle consiste pour une organisation à copier les pratiques adoptées par des firmes performantes et/ou de taille importante. A l'issue d'une étude quantitative (la décision étudiée à trait au choix d'un banquier d'affaire comme conseil lors d'une acquisition), l'existence des trois grandes formes d'imitation interorganisationnelle est vérifiée. Au-delà des trois formes d'imitation mises en lumière par Haunschild et Miner (1997), se dessine la question des raisons amenant des décideurs à imiter leurs concurrents. Pour traiter des logiques mimétiques, il semble nécessaire d'opérer un élargissement des unités d'analyse prise en compte pour se focaliser, non plus seulement sur les organisations, mais aussi sur les individus décideurs. Les approches permettant, à notre sens, d'aborder cette question seront évoquées ci-dessous et mises en perspective par rapport aux modèles de rationalité qu'elles mobilisent (rationalité calculatoire et rationalité basée sur ce qui semble approprié). Les trois logiques mimétiques distinguées dans ce travail (utilitariste, conventionnelle, référentielle) seront ensuite présentées.

\subsection{L'IMITATION, UN COMPORTEMENT RATIONNEL ?}

Le rôle de l'imitation dans la diffusion des innovations avait déjà été souligné par l'économiste autrichien Joseph Schumpeter (1935: 199) : « [L'innovateur] a en quelque sorte vaincu, ouvert un chemin pour d'autres aussi ; il a créé un projet que ces derniers peuvent copier. Ils peuvent le suivre, ils le suivront, d'abord à quelques-uns, puis par masses entières. ». Prolongeant cette conception, des travaux plus récents assimilent l'imitation à une stratégie permettant de réduire la prise de risque associée à l'innovation (Levitt, 1966 ; Schnaars, 1994), de profiter du travail d'«éducation des consommateurs » réalisé par une firme innovante, de diminuer les coûts de recherche et développement (Schnaars, 1994) ou d'apprendre des autres organisations par l'intermédiaire d'un mécanisme de «vicarious learning » (Levitt et March, 1988). Ces travaux, qui s'inscrivent dans une perspective 
$X^{\text {ème }}$ Conférence Internationale de Management Stratégique, Annecy / Genève 13-16 Juin 2006

utilitariste, ont pour point commun de s'intéresser à des formes d'imitation très proches de l'imitation basée sur les résultats décrite par Haunschild et Miner (1997).

Le présupposé utilitariste domine également dans les modèles de Herding Behavior qui appréhendent l'imitation comme un moyen utilisé par les agents pour s'accaparer des informations qui ne sont pas en leur possession (Banerjee, 1992 ; Bikhchandani, Hirshleifer et Welch, 1992) ou qui voient en elle un dispositif permettant de se prémunir contre les retombées négatives que pourraient avoir des décisions malencontreuses (« sharing the blame effect », Scharfstein et Stein [1990]).

En parallèle à ces approches fondées sur des conceptions calculatoires de la rationalité, plusieurs courants de recherche mobilisant des modèles de rationalité alternatifs ont mis l'accent sur les dimensions sociales de l'imitation. C'est notamment le cas de la théorie des conventions qui appréhende des situations dans lesquelles l'incertitude empêche les individus d'agir selon les canons de la rationalité substantive. En présence de tels vides rationnels (Gomez, 1996), il s'agira pour eux de mobiliser un dispositif cognitif collectif, la convention (Favereau, 1989), d'aligner leurs actions sur un modèle considéré comme raisonnable, comme normal (Gomez, 1996 ; Isaac, 2003).

La dichotomie entre une imitation fondée sur le calcul, et une imitation moins « raisonnable », avait déjà été soulignée par Keynes $(1936,2002)$ dans son observation des marchés financiers. Chez Keynes, deux populations d'individus sont clairement distinguées: les investisseurs privés et les investisseurs professionnels. Les premiers sont plongés dans l'incertitude et cherchent à «s'aligner sur le jugement des autres", à se «confronter à l'attitude de la majorité ou de la moyenne »(Keynes, 2002: 250). Les seconds, plus calculateurs tentent de « deviner peu de temps avant le grand public les changements futurs de la base conventionnelle d'évaluation »(Keynes, 1936:170). Si dans les deux cas, les comportements des acteurs sont circonscrits à une imitation basée sur la fréquence (au sens de Haunschild et Miner, 1997), on peut clairement distinguer deux modèles de rationalité. L'un est hérité de la tradition néoclassique, l'autre s'inscrit dans une conception plus hétérodoxe baptisée par Keynes «jugement pratique », proche de la rationalité basée sur « ce qui semble approprié » décrite par March et Olsen (1989).

La rationalité basée sur «ce qui semble approprié », est également au cœur des pressions mimétiques décrites par DiMaggio et Powell (1983). Lorsqu'elles sont confrontées à des problèmes dont les causes sont difficiles à cerner ou dont les solutions sont peu évidentes, les organisations ont tendance à suivre un modèle, souvent une entreprise perçue comme légitime ou très performante (le leader du secteur d'activité par exemple). En outre, comme le 
soulignent notamment Bensedrine et Demil (1998), l'adoption d'une pratique largement diffusée pourra constituer un moyen de se légitimer pour l'organisation.

Parce qu'elle stipule que les organisations imitent des modèles qu'elles considèrent comme légitimes, l'approche néo-institutionnaliste fait appel à une forme d'imitation basée sur les caractéristiques du modèle. Parce qu'elle stipule également que les organisations sont incitées à adopter des pratiques largement diffusées pour se légitimer, cette approche peut également s'inscrire dans une forme d'imitation basée sur la fréquence.

La quête de légitimité sur laquelle nous nous sommes arrêtés serait-elle le pendant organisationnel de la quête d'identité animant les individus ? Telle semble être la conception de Vermeulen et Wang (2005) qui établissent un parallèle entre l'approche néoinstitutionnaliste et une approche ancrée dans le champ de la psychologie sociale, la théorie de l'autocatégorisation. Par le biais d'un mécanisme d'autocatégorisation, les individus sont amenés à se positionner dans leur environnement social, à définir leur groupe social d'appartenance, ou « ingroup» (voir notamment Ashforth et Mael [1989]). Les caractéristiques typiques des membres de l'«ingroup » deviennent alors partie intégrante de l'identité sociale des individus qui le composent (Tajfel et Turner, 1986; Tuner, 1999) ; les individus d'adopter les comportements et les attitudes qui sont, selon eux, caractéristiques de la catégorie sociale à laquelle ils appartiennent. Le mécanisme d'autocatégorisation accentue donc les similarités entre membres d'un même groupe social (Ashforth et Mael, 1989; Turner, 1999). Ce type de comportement ne semble correspondre à aucune des formes d'imitation interorganisationnelle isolées par Haunschild et Miner (1997).

Comme le montrent les développements qui précèdent, les théories qui viennent d'être citées ne s'inscrivent pas toujours dans une même conception de la rationalité. Ces approches diffèrent également dans leurs unités d'analyse (organisation et champ organisationnel pour l'approche néo-institutionnelle, individu et groupe d'individus pour la théorie des conventions et pour la théorie de l'autocatégorisation, individu pour les modèles de Herding Behavior).

\subsection{IMITATION INTEROGANISATIONNELLE ET LOGIQUES MIMETIQUES}

L'imitation interorganisationnelle se pose donc comme un phénomène complexe aux fondements duquel sont imbriqués modèle de rationalité substantive et modèle de rationalité basée sur ce qui semble appropriée; dans lequel se côtoient (parfois au sein d'une même théorie) différents niveaux d'analyse. Sans occulter la diversité des approches théoriques que nous avons présenté, il nous semble que chaque courant comporte une dimension cognitive en ce qu'il projette une ou plusieurs logiques mimétiques sur les individus. 
A l'aide de cette littérature, nous distinguons ici trois logiques mimétiques qui traduisent les dimensions cognitives de l'imitation interorganisationnelle. Nous les avons qualifié de logique utilitariste, logique conventionnelle, logique référentielle. Dans l'esprit de la théorie enracinée (Strauss et Corbin, 2004), l'identification de ces logiques est le résultat d'un processus itératif consistant en un va-et-vient entre théorie et données empiriques.

Basée sur une conception calculatoire de la rationalité, la logique utilitariste est au cœur des comportements décrits par les travaux sur l'innovation. Parce qu'elle consiste en l'imitation d'une pratique en vue de bénéficier de retombées positives liées à son adoption préalable par une ou plusieurs organisations, la logique utilitariste est tournée sur la pratique imitée plus que vers un modèle particulier ou vers un groupe d'organisations indifférenciées.

La logique référentielle est quant à elle tournée vers un modèle et repose sur une conception de la rationalité basée sur ce qui semble approprié. Le modèle pourra être choisi en fonction de caractéristiques communes avec l'organisation imitante (on retrouve ici un mécanisme proche de celui décrit par les théories de l'autocatégorisation) ou, comme chez DiMaggio et Powell (1983), en raison de ses performances, de son statut de «taken-for-granted » au sens de Zucker (1977). Le décideur de voir en l'imitation un moyen d'accroître la légitimité de son organisation. A une échelle individuelle, des mécanismes proches de celui décrit par DiMaggio et Powell (1983) avaient déjà été souligné par Gabriel Tarde (1895) sous le terme « imitation du supérieur par l'inférieur » (l'imitation est supposée permettre aux individus de s'élever dans l'ordre social), ou par certains psycho-sociologues sous le terme « upward comparison » (pour plus de détails, voir Vermeulen et Wang, 2005).

On trouve des manifestations de la logique conventionnelle dans la théorie des conventions, les modèles de herding behavior et, dans une certaine mesure, l'approche néoinstitutionnaliste. En effet, ces travaux ont pour point commun de reposer (de façon non exclusive pour l'approche néo-institutionnaliste) sur des mécanismes cognitifs dans lesquels l'adoption d'une pratique par le groupe sert de justification à l'action individuelle.

Le prisme au travers duquel les données de la recherche sont interprétées peut être synthétisé par le tableau 1. 


\section{Tableau 1 - Les trois logiques mimétiques : récapitulatif}

\begin{tabular}{|l|l|l|l|}
\hline \multicolumn{1}{|c|}{$\begin{array}{c}\text { Logique } \\
\text { utilitariste }\end{array}$} & \multicolumn{1}{|c|}{$\begin{array}{c}\text { Logique } \\
\text { conventionnelle }\end{array}$} & \multicolumn{1}{|c|}{$\begin{array}{c}\text { Logique } \\
\text { référentielle }\end{array}$} \\
\hline Principe & $\begin{array}{l}\text { Imiter une pratique } \\
\text { pour bénéficier de } \\
\text { retombées positives }\end{array}$ & $\begin{array}{l}\text { Reprendre une } \\
\text { solution largement } \\
\text { adoptée autour de soi } \\
\text { pour palier à } \\
\text { l'incertitude }\end{array}$ & $\begin{array}{l}\text { Imiter un ou } \\
\text { plusieurs modèle(s) } \\
\text { en vue d'accroître sa } \\
\text { légitimité ou de } \\
\text { renforcer son identité } \\
\text { sociale. }\end{array}$ \\
\hline Centrée sur & Une pratique imitée & $\begin{array}{l}\text { Un groupe } \\
\text { d'organisations } \\
\text { indifférenciées }\end{array}$ & $\begin{array}{l}\text { Un ou plusieurs } \\
\text { modèle(s) bien } \\
\text { identifié(s) }\end{array}$ \\
\hline Type de rationalité & Calculatoire & $\begin{array}{l}\text { Calculatoire ou basée } \\
\text { sur ce qui semble } \\
\text { approprié }\end{array}$ & $\begin{array}{l}\text { Basée sur ce qui } \\
\text { semble approprié }\end{array}$ \\
\hline $\begin{array}{l}\text { Courants } \\
\text { théoriques }\end{array}$ & $\begin{array}{l}\text { Théorie des } \\
\text { conventions, herding } \\
\text { behavior, approche } \\
\text { néo-institutionnaliste }\end{array}$ & $\begin{array}{l}\text { Approche néo- } \\
\text { institutionnaliste, } \\
\text { théorie de } \\
\text { l'autocatégorisation }\end{array}$ \\
\hline
\end{tabular}

\section{CHAMP ET METHODE DE RECHERCHE}

L'imitation a récemment été au centre de controverses dans le secteur de la radio. Le service proposé par la société Yacast, qui permet aux managers de connaître la liste exhaustive des disques diffusés sur l'antenne de leurs concurrents, a souvent été désigné par les grandes radios musicales comme un instrument facilitant le parasitisme. Ainsi, en février 2004, NRJ (première radio musicale de France en nombre d'auditeurs quotidiens ${ }^{2}$ ) dénonçait dans un communiqué de presse « le clônage de sa programmation » opéré par certaines radios locales et appelait les pouvoirs publics à engager une réflexion «afin que les instruments mis en place pour mesurer la diversité musicale ne deviennent pas, par la livraison à tout le marché de données qui relèvent du secret de fabrication, un instrument d'appauvrissement du pluralisme musical ».

Les tendances mimétiques des radios ont déjà été soulignées par plusieurs recherches menées aux Etats-Unis. Les stations américaines s'inspirent du positionnement adopté par d'autres (Greve, 1996, 1998) ou imitent délibérément des opérations promotionnelles menées par leurs concurrents (Schnaars, 1994 : 11). L'imitation interorganisationnelle, thème de la présente recherche, semble particulièrement courante en radio. Le secteur de la radio constitue donc un

\footnotetext{
${ }^{2}$ Médiamétrie, enquête 126000 Radio, Septembre-Octobre 2005. Critère d'audience cumulée.
} 
terrain d'investigation particulièrement propice à une recherche traitant des logiques mimétiques.

En France, cette industrie est composée de différents types d'organisations. Les anciennes « radios périphériques» (Europe 1, RTL) et les radios du service public cohabitent aujourd'hui avec les radios musicales apparues depuis l'ouverture de la bande FM au début des années quatre-vingt. Ces radios musicales peuvent ne diffuser leurs programmes que sur une petite partie du territoire français (radios locales et régionales) ou avoir une dimension nationale. Les radios musicales nationales sont souvent désignées par le terme « réseaux ».

Dans les radios musicales, le mode de fabrication du programme est extrêmement standardisé (Ahlkvist et Fisher, 2000) : il consiste en la définition d'une playlist (liste de disques diffusés quotidiennement) et en l'assignation d'un nombre de passages quotidiens à chaque titre. Chaque station établit sa playlist en fonction de sa cible et de son positionnement musical (le format). On pourra distinguer le format «Top 40 » (adopté par NRJ et de très nombreuses radios locales) qui consiste à diffuser les succès du moment («hits »), de formats plus thématiques (Rap R\&B / Pop Rock / Rock / Dance...). Malgré les différences de formats, il n'est cependant pas rare de voir un même titre diffusé sur de très nombreuses radios. Comme l'explique Christian Blachas (2004) : «La différence se fait sur des notions ténues : musique du début des années 80, du milieu des années 80, de la fin des années 80. [...] Toujours les mêmes tubes, les mêmes standards. Et toujours cette dictature de la playlist qui enlève à la radio la spontanéité qui est pourtant sa raison d'être ». En France, les radios musicales ont l'obligation légale de réserver une place importante à des titres francophones dans leurs programmes (trois formules existent ; 40\% de titres francophones dont $20 \%$ de nouveaux talents; $35 \%$ de titres francophones dont $25 \%$ de nouveaux talents; $50 \%$ de titres francophones dont $15 \%$ de nouveaux talents ${ }^{3}$ ).

Des instruments de «recherche musicale » sont parfois utilisés pour limiter les risques liés à l'entrée d'un nouveau titre en playlist. Le « call-out » est une enquête effectuée par téléphone sur un panel d'auditeurs représentatif du public de leur station. L' « auditorium » consiste à rassembler des auditeurs pour leur faire évaluer les différents éléments qui composent le programme (Ahlkvist et Fisher, 2000).

L'outil Yacast offre aux radios abonnées la possibilité d'accéder, d'une part, à des classements thématiques réalisés sur la base de relevés exhaustifs des disques diffusés sur une

\footnotetext{
${ }^{3}$ Loi du 30 septembre 1986 modifiée. Lettre du Conseil Supérieur de l'Audiovisuel n ${ }^{\circ} 13$, 1999. Un «nouveau talent » est un artiste ayant entamé sa carrière après 1974 et n'ayant pas encore obtenu deux disques d'or sur deux albums distincts.
} 
trentaine de radios nationales et locales et, d'autre part, à la liste exhaustive des disques diffusés par ces radios (les disques sont alors classés en fonction de leur fréquence de passage). La plupart des radios musicales, nationales comme locales, bénéficient aujourd'hui de ce service et ont ainsi quotidiennement accès aux programmations musicales de leurs concurrents, qu'elles peuvent imiter d'autant plus facilement.

Le rapport sur la diversité musicale dans le paysage radiophonique établi en 2003 par l'Observatoire de la Musique souligne un «effet d'endogamie » dans les programmations musicales des radios du panel Yacast. En 2003, 19 des 31 radios observées avaient en commun au moins $50 \%$ de leur playlist ${ }^{4}$. Le phénomène semble particulièrement marqué chez les radios destinées aux cibles jeunes et jeunes adultes.

\section{CHOIX METHODOLOGIQUES}

Résolument interprétative, cette recherche vise à améliorer la compréhension des logiques en vigueur lorsque des décideurs imitent d'autres organisations. Le terrain d'étude est composé d'un seul secteur: les radios musicales françaises. Nous ne nous intéresserons qu'à un seul type de décision : l'entrée en programmation d'un nouveau disque. Les décideurs interrogés occupent la fonction de Directeur des Programmes ou de la Programmation Musicale et décident seuls (le plus souvent) des entrées en programmation.

La collecte des données primaires a donné lieu à une série de vingt entretiens semi-directifs réalisés dans des réseaux nationaux et des radios locales entre mai 2004 et octobre 2005. Les premières questions (phase introductive) concernent les fonctions occupées par le répondant, son parcours dans l'entreprise, le format musical et la cible de sa radio. Le reste de l'entretien consiste en l'écoute de plusieurs extraits musicaux (d'une trentaine de secondes chacun) issus de la programmation musicale de la radio visitée. En moyenne, dix extraits sont soumis à l'écoute du répondant pour chaque entretien.

Après l'écoute de chaque extrait, il est demandé au répondant de raconter l'historique du titre dans la programmation de sa station et d'expliquer les raisons de son entrée en playlist. Les titres proposés à l'écoute sont choisis parmi les disques les plus fréquemment diffusés par la station et ont été entrés dans la programmation au cours des semaines précédant l'entretien. Des relances sont effectuées (à partir d'un guide d'entretien) lorsque les thèmes de la recherche sont évoqués spontanément par le répondant (imitation, mimétisme, concurrents, relations avec les maisons de disques, doutes/incertitudes, etc.)

\footnotetext{
${ }^{4}$ Observation réalisée sur la base du Top 40 des radios du panel Yacast. Rapport disponible sur http://rmd.citemusique.fr/observatoire/
} 
Initialement, le dispositif a été imaginé pour éviter au répondant d'avoir à décider lui-même des titres à évoquer en entretien et pour l'obliger à éteindre la radio (souvent allumée au moment de la rencontre) de façon à faciliter le travail de retranscription. Ce «protocole » semble avoir trois avantages qui n'avaient pas été prévus initialement : (1) son caractère ludique favorise la mise en confiance du répondant et évite une certaine lassitude, (2) il sollicite la mémoire auditive, particulièrement développée chez les professionnels de la radio, (3) il rappelle le déroulement d'une réunion de travail ordinaire entre un responsable de la programmation et un chargé de promotion d'une maison de disque.

Chaque entretien est enregistré puis intégralement retranscrit. Une analyse de contenu thématique est pratiquée à l'aide du logiciel N-Vivo. Les trois phases de codages reprennent le processus décrit par Richards (2005) : codage signalétique, codage descriptif (en fonction des sujets évoqués par le répondant), codage analytique (semi-émergent, reprise des trois grandes logiques détaillées plus tôt).

La connaissance du secteur de la radio et de ses enjeux est enrichie par des entretiens de contexte menés de façon formelle ou informelle auprès d'intervenants du secteur (animateurs, programmateurs) ou de la filière musicale (chargés de promotion des maisons de disques notamment) et par des données secondaires. Ces dernières peuvent être fournies par les répondants eux-mêmes (documents de travail, e-mails envoyés par les chargés de promotion des maisons de disques), glanées dans la presse économique, généraliste et professionnelle, sur les sites Internet des instituts de mesure de l'audience radio, recueillies lors de salons professionnels (conférences du salon annuel Le Radio!), etc. Les principaux résultats de la recherche ont été communiqués aux répondants qui en ont formulé la demande.

\section{LES TROIS LOGIQUES MIMETIQUES A L'OEUVRE}

Comme attendu, l'imitation est une pratique extrêmement généralisée en radio. Ce constat est d'ailleurs partagé par les chargés de promotion des maisons de disques rencontrés dans le cadre des entretiens de contexte. L'extrait suivant est tiré d'un de ces entretiens.

«Je pense que les programmateurs sont très attentifs à ce qu'on leur dit, euh... il suffit qu'ils l'entendent eux sur une autre radio pour se dire "en fait ouais..." Ca marche bien ça. Ils sont très attentifs à ce que font les autres radios. Parfois quand on doute, il suffit de l'entendre sur une autre radio pour se dire "en fait ça passe super bien, je devrais m’y mettre." »

Les répondants ne semblent éprouver aucune difficulté à évoquer le thème de la recherche. $\mathrm{Au}$ total, deux cent sept extraits ont été proposés. Dans plus d'un tiers des cas, les répondants 
expliquent avoir, pour une raison ou pour une autre, imité un ou plusieurs de leurs concurrents.

L'imbrication des niveaux d'analyse individu et organisation qui caractérise la littérature sur l'imitation interorganisationnelle se retrouve sur le terrain. Comme le montrent les extraits suivants, les répondants interrogés se définissent souvent par le nom de la station qu'ils dirigent. Inversement, lorsqu'ils évoquent une station concurrente, ils peuvent indifféremment la désigner par son nom ou par le nom de la personne qui en assure la programmation musicale.

«Si je disais que c'est ma radio qui a lancé Calogero, ça serait très prétentieux mais le premier qui a osé passer un titre de Calo sur l'ancien album... pas le précédent mais encore un autre avant, c'est moi !»

«Quand tu regardes... NRJ a bien travaillé au début de la saison, en septembre-octobre, Roberto [le directeur général des programmes et de la marque NRJ] a bien bossé avec Madonna... »

$\mathrm{Au}$ sein d'une même logique, plusieurs variations peuvent être observées: nous ne détaillerons ici que les formes les plus fréquentes. Elles seront illustrées à l'aide de citations issues des retranscriptions. Dans l'optique interprétativiste qui est la notre, ces citations seront discutées à l'aide d'éléments théoriques.

\subsection{LOGIQUE UTILITARISTE}

La logique utilitariste renvoie à l'imitation d'une pratique afin de bénéficier des retombées positives liées à l'adoption préalable du titre par une ou plusieurs autres organisations.

\subsubsection{Profiter des tests mis en place par les concurrents}

L'imitation peut permettre aux organisations d'économiser sur des coûts liés à l'innovation. Nous avons évoqué les outils de «recherche musicale » mis en place par certaines radios en vue de tester les disques qu'elles diffusent. En raison de leur coût élevé, ces outils sont réservés aux réseaux nationaux et aux radios régionales les plus puissantes. Comme le montrent les verbatims suivants, l'imitation peut constituer, pour les radios locales, un bon moyen de s'approprier les résultats de ces « tests ».

«On n'a pas les moyens d'une radio comme... des réseaux. Donc on ne peut pas travailler de la même façon. Alors on peut travailler comme le font certaines radios locales, c'est-à-dire d'attendre et de voir ce que font les autres en se disant voilà, il y a un organisme qui s'appelle Yacast qui fait la pige de toutes les radios, il suffit de regarder le Top 40 des radios qui testent et puis, on sait très bien que s'il est numéro un, ça veut dire que ça teste. Bon, voilà! C'est facile. » 
«Il y a des morceaux comme Pink, Get the party started, je n'en peux vraiment plus. Mais... je m'aperçois que c'est toujours à fond sur NRJ, c'est toujours à fond sur Fun donc je me dis que ça plait toujours aux auditeurs. Si le titre était vraiment burné ${ }^{5}$ à $100 \%$, ils le baisseraient je pense. Donc je suis encore bon. »

Ce parasitisme est facilité par la généralisation du service proposé par Yacast. Les radios mises sur écoute semblent faire l'objet d'une attention particulière de l'ensemble des acteurs du secteur. On retrouve ici une conclusion déjà formulée par Greve (1998) qui souligne une condition sine qua non à l'imitation d'une pratique : l'observabilité.

La démarche consistant à imiter les radios « qui testent » pour avoir l'assurance de diffuser les disques qui suscitent le plus fort engouement chez les auditeurs avait été dénoncée par NRJ, leader du secteur, qui s'estimait «plagiée et pillée dans ses méthodes de programmation et son savoir-faire $»^{6}$. Si cette démarche semble propre aux radios locales, d'autres manifestations de la logique utilitariste peuvent également être relevées dans les discours des Directeurs des Programmes et/ou de la Programmation Musicale de grands réseaux nationaux.

\subsubsection{Profiter du travail de développement réalisé par les autres}

L'imitation peut permettre de profiter du travail de développement réalisé par un ou plusieurs concurrents. Cette remarque, déjà formulée par Levitt (1966) dans sa métaphore de la pomme juteuse ${ }^{7}$ s'applique particulièrement à l'industrie étudiée.

«Sur des choses un petit peu tendancieuses ou des choses un peu moins faciles, je pense que c'est plus à NRJ de déblayer le terrain. Eux ont une force de frappe qui est beaucoup plus... et après nous on y vient. »

Ce type d'attitude semble renforcé par la croyance selon laquelle seule la diffusion des plus gros succès du moment (les « hits ») peut permettre à la radio de rencontrer le succès. Cette croyance, particulièrement développée chez les programmateurs des radios généralistes, constitue le fondement du format « Top $40 »$.

« Le but du jeu, c'est qu'à n'importe quel moment de la journée, l'auditeur lambda qui va se brancher, il ait son hit. Et ça, c'est impératif! Si dans le quart d'heure, il n'a pas son hit, vous êtes mort! Vous êtes mort! Le mec, il n'a pas son hit au moment où il branche la radio, il va sur la concurrence. »

\footnotetext{
${ }^{5}$ Un titre « burné » est un titre que les auditeurs n'ont plus envie d'entendre.

${ }_{7}^{6}$ Communiqué de presse NRJ, 9 février 2004.

${ }^{7}$ "You don't have to get the first bit on the apple to make out. The second or third juicy bit is good enough. Just be careful not to get the tenth skimpy one » (Levitt, $1966: 66)$.
} 
L'innovation apparaît alors, à la fois comme une démarche nécessaire au renouvellement des "playlists » et comme une opération extrêmement risquée. Les programmateurs sont alors tentés de laisser aux autres le soin d'innover.

« Tragédie ${ }^{8} \ldots$ quelle tragédie ! Là aussi, on n'était pas très convaincus. Donc on a laissé filer un petit peu. En se disant : "On va voir si vraiment, c'est aussi tubesque qu'on nous l'annonce." Laisser filer c'est laisser construire. On ne peut pas tout démarrer, c'est évident. Comme aucune radio ne démarre tout... c'est des titres qui démarrent par telle ou telle radio. Là en l'occurrence, le titre il a démarré, je crois, par Fun. Qui a fait un bon choix puisque ça a été un gros tube !»

La tentation est encore plus forte lorsque la décision porte sur un titre éloigné du format initial de la radio. L'imitation devient alors un moyen de s'immuniser contre les retombées négatives (en termes de perte d'audience) que pourrait avoir l'entrée du disque en playlist. C'est notamment le cas pour ce directeur des programmes d'un important réseau régional thématique, qui par la législation sur les quotas de chanson française, se voit contraint de sortir de son positionnement musical habituel.

«Ca c'est Amine. La question c'est pourquoi on le joue ? C'est ça ? Tout simplement parce qu'en France, il y a une loi qui s'appelle euh... la loi des quotas. Et c'est compliqué parce que la musique qu'on diffuse, en général, elle n'a pas une grosse expression francophone. Pour ce type de morceau, on est beaucoup moins starters. Parce que comme c'est pas "format", je ne vais pas m'amuser à jouer en avance un morceau qui n'est pas ce que mes auditeurs attendent. J'attends que ça passe sur d'autres radios. C'est juste le fait que quand les gens vont l'entendre chez moi, s'ils ne l'aiment pas et qu'ils vont sur une autre radio ils risquent de tomber aussi dessus. Donc au bout d'un moment, ils vont peut-être s'habituer ou se dire: "Moi j'aimais pas mais eux ils le passent aussi donc je vais revenir sur ma radio." "

Comme le souligne Schnaars (1994), ce type de démarche constitue souvent une pièce maîtresse de la stratégie des leaders. Dans le secteur de la micro-informatique, il n'est pas rare de voir Microsoft laisser à des entreprises naissantes le soin de supporter les risques associés à l'innovation pour s'approprier, en cas de succès, leurs découvertes par des opérations de croissance externe.

\subsection{LOGIQUE CONVENTIONNELLE}

La logique conventionnelle renvoie à l'adoption d'une pratique déjà adoptée par une majorité d'organisations. Elle s'inscrit dans une procédure régulière de résolution d'un problème lié à une situation d'incertitude : une convention (Gomez, 1996).

\footnotetext{
${ }^{8}$ Le groupe Tragédie a rencontré le succès en 2004, notamment avec ses titres « Hey ho ! » et « Sexy pour moi ».
} 
$X^{\text {ème }}$ Conférence Internationale de Management Stratégique, Annecy / Genève 13-16 Juin 2006

\subsubsection{Face à l'incertitude, s'aligner sur la solution adoptée par la majorité}

L'état d'incertitude se caractérise par l'impossibilité laissée aux individus de recourir à un quelconque raisonnement probabiliste pour émettre des prévisions. Cette description semble parfaitement s'appliquer à la tâche du programmateur qui consiste à prévoir, à partir des envois réalisés par les maisons de disques, les titres qui susciteront quelques mois plus tard l'engouement des auditeurs.

Les outils de «recherche musicale » ne deviennent alors que des instruments de validation et de vérification a posteriori. Leur potentiel prospectif est des plus limités.

« Ce sont des phénomènes assez extraordinaires parce que des titres comme ça il y en a eu une flopée. Pourquoi est-ce que c'est celui-là qui a plu ? Bon, c'est ça la musique! C'est ce que l'on explique pas toujours, parce que tout n'est pas explicable, tout n'est pas mathématique, tout n'est pas scientifique. »

Quels seront les succès futurs? Quels sont les disques qui sont susceptibles de plaire au public ? Comment trier le bon grain de l'ivraie ? Face à une telle situation d'incertitude, l'imitation des autres devient plus efficace que la recherche d'une solution personnelle (le problème initial étant de toutes façons insoluble).

« Je reçois, je sais que c'est un tube... et boum. Tu le sais... c'est un tube !

$\mathrm{Tu}$ vois que des radios le rentrent en province, que NRJ aussi, derrière. Tu sais que c'est un tube... TU LE SAIS! Je vais voir mon DG, je lui dis "ça c'est un tube !" "

L'adoption par autrui permet, a priori, d'avoir une idée du succès futur du disque auprès des auditeurs. Il devient également, a posteriori, à la fois un critère de rationalisation du choix effectué et le principal indicateur du succès du titre.

« Maintenant c'est un gros carton parce que toutes les radios le jouent. »

Le raisonnement est tautologique : un titre est voué à devenir un tube car il est diffusé par d'autres radios, un titre ayant été diffusé par d'autres radios est un tube. Nous retrouvons ici la prophétie autoréalisatrice décrite par Keynes (1936) et reprise par Gomez (1996).

\subsubsection{Instrumentaliser la convention}

Le problème initial : «ce disque va-t-il plaire au public ?» est écarté à défaut d'avoir été résolu. Le jeu du programmateur se transforme progressivement en jeu de coordination (Schelling, 1960) dans lequel «il s'agit de choisir X si l'autre choisit X et Y si l'autre choisit $Y »($ Batifoulier et Larquier, 2001 : 105), de deviner quels seront les disques 
adoptés par les concurrents. L'alignement maximum sur la convention devient, plus qu'un moyen de se différencier des concurrents, un facteur de réussite.

"L'idéal en prog, c'est de jouer les "bons" titres, plus que les autres. On se dit: "Pourquoi les radios passent autant de fois les disques?", c'est simplement parce que, quand tu as un disque qui cartonne, tout le monde le joue... et que pour avoir une chance d'être plus fort que les autres, il faut le jouer plus que les autres. »

A la manière des spéculateurs dépeints par Keynes (1936), certains programmateurs semblent avoir instrumentalisé la convention et cherchent à deviner, un peu à l'avance, les futurs points d'alignement.

«Moi j'ai pris les devants, j'ai essayé de savoir quel serait le prochain single pour ne pas non plus être en décalage. L'avantage, c'est que je l'ai joué en nouveauté quand les autres ne le jouait pas encore. C'était une façon d'être un peu en avance MAIS, pas non plus d'être TROP en avance. »

Si cette démarche ne s'inscrit pas dans la définition de l'imitation présentée plus haut (il n'y a pas adoption préalable de la pratique par un modèle), elle constitue néanmoins le prolongement extrême de la logique conventionnelle lorsqu'elle est mue par une rationalité calculatrice.

\subsection{LOGIQUE REFERENTIELLE}

La logique référentielle renvoie à l'imitation d'une pratique adoptée par un référent (une organisation bien identifiée). Elle s'articule avec la quête de légitimité des organisations et la recherche d'une identité sociale positive des individus.

\subsubsection{Imiter une organisation considérée comme légitime}

Comme chez DiMaggio et Powell (1983), la légitimité du modèle semble parfois jouer un rôle déterminant dans les comportements mimétiques des programmateurs radios. C'est notamment le cas chez ce Directeur des Programmes d'un réseau régional généraliste qui explique avoir attendu qu'un disque soit entré en programmation sur Skyrock pour l'adopter :

«Lemar, je vais être honnête avec vous, euh... on avait reçu un premier single il y a quelques temps et... dans ce cas précis, j'ai attendu de savoir quelles seraient les réactions sur les autres radios. En fait j'avais vraiment envie de savoir comment une radio comme Skyrock allait réagir. Je me demandais... je me disais : " Bon, ça fait très Motown, c'est sur une cible plutôt adulte ». Je voulais vraiment savoir comment une radio comme Skyrock allait ce positionner : est-ce que ce titre là allait leur plaire ? Allait plaire aux jeunes ou rester sur une dynamique de jeunes adultes? Skyrock est une radio jeune, donc je voulais savoir si les jeunes qui écoutent Skyrock allaient aimer le morceau. L'histoire a fait que ça marche plutôt pas mal 
chez eux aussi. NRJ l'a rentré, Skyrock derrière, en plus à forte rotation.

Donc on s'est dit qu'il fallait y aller. »

Le modèle de légitimité mobilisé semble néanmoins différer de celui décrit par Zucker (1977), DiMaggio et Powell (1983). Comme l'explique Dejean (2005 : 169) : «La légitimité cognitive repose sur la connaissance des acteurs », «Une organisation acquiert une légitimité cognitive lorsqu'elle obtient le statut de "taken-for-granted", son existence est alors considérée par les autres organisations comme allant de soi. » Dans l'extrait précédant, le répondant ne semble pas attribuer le statut de «taken-for-granted» à son référent. La légitimité attribuée à Skyrock est plus sélective et se limite à un domaine particulier : les jeunes auditeurs. Certaines radios seront considérées comme légitimes sur d'autres critères. C'est notamment le cas des réseaux thématiques Europe 2 et Fun Radio qui bénéficient d'un certain crédit sur leur genre musical de prédilection (respectivement le Rock et la Dance).

« C'est le rock qui m'intéresse sur Europe 2. Au niveau de Fun, euh... c'est plutôt la Dance qui m'intéresse parce que c'est "Fun Radio Groove \& Dance". NRJ, c'est une radio généraliste donc [...] on regarde [plutôt] les autres. »

Ce mécanisme est connu des théories de l'identité sociale sous l'appellation « relatedattributes similarity » (pour plus de détails, voir Vermeulen et Wang, 2005). Les individus comparent leurs actions dans un domaine particulier (diffuser un disque susceptible de plaire aux jeunes ou diffuser un disque d'un genre musical particulier) avec des caractéristiques reliées à ce domaine (ici, la cible ou le format).

\subsubsection{Anéantir son concurrent en devenant son double}

Plus surprenant, certains répondants s'acharnent à stigmatiser les modèles qu'ils imitent. Au palmarès des radios les plus souvent conspuées, NRJ, le leader du secteur occupe incontestablement la première place.

Alors ça... je vais pas te mentir. Je déteste. Quand on me l'a amené, je me suis dit : "Le mec chante du nez, c'est la danse des canards, j'en veux pas !" Euh... je vais pas faire le show... on l'a rentré genre deux mois après NRJ. [...] Bon, bref, ce truc là faut le rendre à NRJ. Je trouvais ça tellement inintéressant et pauvre artistiquement que... voilà. [Un peu plus tard dans l'entretien] Mais ça m'embête. Les gens, j'ai du mal à comprendre qu'ils aiment les sous trucs. Remarque, on ne peut pas reprocher à NRJ de ne pas avoir de personnalité... c'est de la soupe mais ça a de la gueule !

Pour comprendre ce dualisme répulsion-imitation, on pourra se référer aux travaux de René Girard (1972) qui place l'imitation dans le contexte de la crise sacrificielle. Le «désir 
mimétique » décrit par Girard découle d'une soif de violence innée chez l'être humain : imiter l'autre, c'est l'anéantir, en devenir le double monstrueux.

\subsubsection{Imiter des organisations qui nous ressemblent}

L'autocatégorisation, un autre mécanisme bien connu en psychologie sociale et déjà évoqué plus haut, apparaît également de façon récurrente dans les entretiens. Les répondants ont ainsi tendance à reprendre des solutions adoptées par des organisations qu'ils catégorisent dans leur groupe social. Le répondant interrogé dans le verbatim suivant est Directeur des Programmes d'un réseau régional membre du GIE « Les Indépendants » qui rassemble plus d'une centaine de radios locales et régionales au sein d'une offre publicitaire commune. Les radios qu'il cite sont toutes membres du groupement. L'appartenance au GIE « Les Indépendants » renvoie à un fort sentiment identitaire qui justifie l'imitation.

«Mario... ce sont les résultats aux Etats-Unis qui m’ont intéressé. C'est-àdire que c'était numéro un aux Etats-Unis. Et puis en regardant sur Yacast, et en regardant une radio comme Ado par exemple, je me suis aperçu que c'était à fond chez eux. Et je me suis dit : "Bruno W., il est à bloc là-dessus, et je pense qu'il a tout compris, donc c'est très bien". Ado, de temps en temps je jette un œil. Scoop, Ado, Voltage, Vibra, Alouette, Hit West de temps en temps, parce qu'ils sont un peu comme nous. »

\section{DISCUSSION}

Par cet article, nous avons souhaité améliorer la connaissance de l'imitation interorganisationnelle en complétant les travaux existants par une investigation empirique sur le thème des logiques mimétiques. Comme l'indique Haunschild (1993), savoir comment les organisations s'imitent et savoir pourquoi les organisations s'imitent renvoient à deux questions différentes.

Les approches théoriques existantes en matière d'imitation interorganisationnelle proposant des explications diverses, nous avons délibérément adopté une démarche théorique fondée sur le pluralisme. Nous n'envisageons pas cette diversité théorique comme le point de départ à une démarche hypothético-déductive visant à tester ces approches : nous avons cherché ici à construire un prisme au travers duquel interpréter les données qualitatives recueillies dans le cadre de la recherche.

Les différentes approches théoriques mobilisées ici s'intéressent à un aspect particulier de l'imitation pour servir un projet de recherche beaucoup plus vaste. Les pressions mimétiques qu'étudient les néo-institutionnalistes ne rassemblent, par exemple, qu'une partie des facteurs générateurs d'isomorphisme institutionnel (Mizruchi et Fein, 1999). De même, la théorie des conventions n'est pas qu'une simple théorie des comportements mimétiques (Gomez, 1996). 
Néanmoins, aussi vastes et générales soient-elles, ces approches proposent chacune une «théorie » de l'imitation interorganisationnelle en ce qu'elles postulent des logiques mimétiques chez les individus. Leur croisement et leur mise en perspective par rapport à notre matériau empirique nous ont permis de formuler notre typologie.

Les apports de cet article sont, selon nous, les suivants. D’un point de vue théorique, différentes approches ont été articulées. Trois logiques (utilitariste, conventionnelle, référentielle), qui ne mobilisent pas les mêmes formes de rationalité ont ainsi été identifiées et nommées. Certains courants théoriques auxquels nous avons fait appel ont été peu mobilisés par les recherches en management abordant la question de l'imitation interorganisationnelle. C'est notamment le cas de la théorie de l'autocatégorisation, souvent mise de coté au profit des approches néo-institutionnalistes, qui traduit la dimension psycho-sociale de l'imitation. Cette orientation nous a également permis de sortir du paradigme «technico-rationnel » qui caractérise la plupart des travaux sur la programmation musicale des radios (Ahlkvist, 2001). D’un point de vue empirique, les trois logiques ont été observées de façon détaillée dans un secteur jusqu'ici peu étudié par les chercheurs en sciences de gestion francophones. Les acteurs de terrain auxquels ont été communiqués les résultats de cet article ont semblé intéressés par le détail de chaque logique et certains chargés de promotion de l'industrie musicale ont ici trouvé des éléments pouvant leur permettre d'amorcer une réflexion visant à améliorer l'efficacité de leurs actions de promotion.

Le principal apport de notre travail tient à l'observation de ces trois logiques de façon concomitante dans l'industrie étudiée. Ce résultat semble indiquer que les approches théoriques actuelles ne proposent qu'une image tronquée de l'imitation interorganisationnelle en ne discutant du phénomène «que de façon amputée» ou en se focalisant «sur des situations dans lesquelles une seule de ces logiques domine ${ }^{9} . »$

En ne s'intéressant qu'à des formes d'imitation interorganisationnelle basées sur les résultats, les travaux sur l'innovation ne surestiment-ils pas le rôle de la logique utilitariste ? Comme le laisse entendre Romelaer (1999), la théorie des conventions ne réduit-elle pas son champ d'analyse en se focalisant sur des situations d'incertitudes radicales ? Plus généralement, les logiques mimétiques ne sont-elles pas contingentes aux comportements dans lesquelles elles s'inscrivent et aux situations d'incertitude qu'elles permettent de surmonter? Pour creuser ce point, un effort de recherche visant à articuler formes d'imitation (au sens de Haunschild et

\footnotetext{
${ }^{9}$ Ces remarques ont été émises par un évaluateur anonyme que l'auteur tient à remercier tout particulièrement.
} 
Miner, 1997), logiques mimétiques et situations d'incertitude semble indispensable. La typologie formulée dans cet article pourra être mobilisée dans une telle démarche.

Plusieurs critiques peuvent nous être adressées. Le choix d'un secteur atypique, s'il semble intéressant dans la thématique de la recherche, permet de poser la question de la transposition possible des résultats à d'autres contextes. Le recours à des données qualitatives purement déclaratives, s'il semble inévitable compte tenu de l'orientation cognitiviste de ce projet, laisse entrevoir la possibilité de compléter notre corpus par des données quantitatives non soumises au biais de subjectivité (telles que les données Yacast, basées sur des relevés exhaustifs de programmation) et permettant d'ouvrir l'analyse à des éléments plus concrets.

Enfin, une limite inhérente à l'orientation générale de cet article a été soulignée par plusieurs professionnels réagissant aux résultats qui leur ont été communiqués. Tout en se reconnaissant dans les descriptions détaillées plus haut, ces derniers soulignent l'importance, pour une station, de ne pas être la copie conforme de ses concurrents. Le passage suivant est extrait d'un mail envoyé par le directeur des programmes d'une importante station régionale.

« Il manque juste cette petite pointe de particularité propre à chaque station de manière proportionnelle aux convictions de chacun. »

Cette remarque tend à corroborer l'analyse de Vermeulen et Wang (2005) selon laquelle le besoin d'être similaire et le besoin d'être différents sont tous les deux au cœur des phénomènes de gestion.

On pourra ici établir un parallèle entre la théorie des conventions dans laquelle le mimétisme des individus permet de passer outre les situations d'incertitude en générant des convictions et le propos cité plus haut qui évoque les «convictions de chacun» pour expliquer « cette petite pointe de particularité propre à chaque station ».

A la lumière des développements qui précèdent, il convient de s'interroger sur l'interaction possible entre une imitation génératrice de convictions et une différenciation fondée sur ces mêmes convictions. Imitation et différenciation ne constituent-elles pas les deux faces de Janus d'un même phénomène cognitif? 


\section{BIBLIOGRAPHIE}

Ahlkvist, J. A., « Programming philosophies ant the rationalization of music radio ", Media, Culture \& Society, Vol. 23, p. 339-358, 2001.

Ahlkvist, J. A. et Fisher, G., « And the hits just keep on coming: Music programming standardization in commercial radio », Poetics, Vol. 27, p. 301-325, 2000.

Ashforth, B. E. et Mael, F., "Social Identity Theory and the Organization », The Academy of Management Review, Vol. 14, No. 1, p. 20-39, 1989.

Banerjee, A. V., «A Simple Model of Herd Behavior », The Quaterly Journal of Economics, Vol. 107, No. 3, p. 797-817, 1992.

Batifoulier, P. et Larquier, G. d., " La convention en théorie des jeux », dans P. Batifoulier, Théorie des conventions, Economica, p. 99-126, 2001.

Baudonnière, P.-M., Le mimétisme et l'imitation, Flammarion, 1997.

Bensedrine, J. et Bemil, B., "L'approche néo-institutionnelle des organisations », dans H. Laroche et J.-P. Nioche, Repenser la stratégie, fondements et perspectives, Institut Vital Roux, Vuibert, p. 85-110, 1998.

Bikhchandani, S., Hirshleifer, D. et Welch, I., " A Theory of Fads, Fashion, Custom, and Cultural Change as Informational Cascades ", The Journal of Political Economy, Vol. 100, No. 5, p. 992-1026, 1992.

Blachas, C., « Le show ou le froid », CB News, N 803, p. 4, 27 septembre-3 octobre 2004.

Dejean, F., L'investissement socialement responsable : Etude du cas français, Vuibert (Fnege), 2005.

DiMaggio, P. J. et Powell, W. W., « The Iron Cage Revisited: Institutional Isomorphism and Collective Rationality in Organizational Fields "), American Sociological Review, Vol. 48, No. 2, p. 147-160, 1983.

Ellsworth, F., « The Concept of Imitation », The American Journal of Sociology, Vol. 32, No. 3, p. 367-378, 1926.

Favereau, O., « Marchés internes, marchés externes », Revue Economique, Vol. 40, No. 2, p. 273-328, 1989.

Girard, R., La violence et le sacré, Bernard Grasset, Paris, 1972.

Gomez, P.-Y., Le gouvernement de l'entreprise, InterEditions, Paris, 1996.

Greve, H. R., " Patterns of Competition: The Diffusion of a Market Position in Radio Broadcasting », Administrative Science Quaterly, Vol. 41, No. 1, p. 29-60, 1996.

Greve, H. R., « Managerial Cognition and the Mimetic Adoption of Market Positions: What You See is What You Do ", Strategic Management Journal, Vol. 19, No. 10, p. 967-988, 1998.

Greve H.R., " Interorganizational Learning and Heterogeneous Social Structure ", Organization Studies, Vol. 26, No. 7, p. 1025-1047, 2005.

Haunschild, P. R., " Interorganizational Imitation: The Impact of Interlocks on Corporate Acquisition Activity », Administrative Science Quaterly, Vol. 38, No. 4, p. 564-592, 1993.

Haunschild, P. R. et Miner, A. S., « Modes of Interorganizational Imitation: The Effect of Outcome Salience and Uncertainty », Administrative Science Quaterly, Vol. 42, No. 3, p. 472500, 1997. 
$X^{\text {ème }}$ Conférence Internationale de Management Stratégique, Annecy / Genève 13-16 Juin 2006

Haveman, H. A., "Follow the Leader: Isomorphism and Entry Into New Markets », Administrative Science Quaterly, Vol. 38, No. 4, p. 593-627, 1993.

Isaac, H., "Paradoxes et conventions ", dans V. Perret et E. Josserand, Paradoxes et organisations, Ellipses, p. 147-163, 2003.

Johnson, G., Scholes, K., Whittington, R. et Fréry, F., Stratégique (7ème édition), Pearson Education, 2005.

Keynes, J. M., Théorie Générale de l'Emploi, de l'Intérêt et de la Monnaie, Payot, 1969 (édition originale 1936).

Keynes, J. M., « La théorie générale de l'emploi », dans J. M. Keynes, La pauvreté dans l'abondance, Gallimard, p. 240-260, 2002.

Levitt, T., «Innovative Imitation », Harvard Business Review, Vol. 74, No. 5, p. 63-70, 1966.

Levitt, B. et March, J. G., « Organizational Learning », Annual Review of Sociology, Vol. 14, p. 319-340, 1988.

March, J. G. et Olsen, J. P., Rediscovering Institutions: The Organizational Basic of Politics, Free Press, New York, 1989.

Mizruchi, M. S. et Fein, L. C., « The Social Construction of Organizational Knowledge: A Study of the Uses of Coercive, Mimetic, and Normative Isomorphism ", Administrative Science Quaterly, Vol. 44, No. 4, p. 653-683, 1999.

Richards, L., Handling Qualitative Data: A Practical Guide, Sage Publications, 2005.

Romelaer, P., « Quelques problèmes de la théorie des conventions », Les cahiers de l'Artemis, Vol., No. 2, p. 149-208, 1999.

Scharfstein, D. S. et Stein, J. C., « Herd Behavior and Investment», The American Economic Review, Vol. 80, No. 3, p. 465-479, 1990.

Schelling, T., The Strategy of Conflict, Harvard University Press, Cambridge, 1960.

Schnaars, S. P., Managing Imitation Strategies, The Free Press, 1994.

Schumpeter, J., Théorie de l'évolution économique, Dalloz, 1999 (édition originale 1935).

Strang D. et Soule S.A., « Diffusion in Organizations and Social Movements : From Hybrid Corn to Poison Pills », Annual Review of Sociology, Vol. 24, p. 265-290, 1998.

Strauss, A. et Corbin, J., Les fondements de la recherche qualitative, Academic Press Fribourg, 2004.

Tajfel, H. et Turner, J. C., "The Social Identity Theory of Intergroup Behavior », dans S. Worchel et W. G. Austin, Psychology of Intergroup Relations, 2nd edn., Nelson-Hall, Chicago, 1986.

Tarde, Les lois de l'imitation (2ème édition), Bibliothèque Paul-Emile-Boulet de l'Université du Québec à Chicoutimi, 1895.

Turner, G., " Some Current Issues in Research on Social Identity and Self-categorization Theories », dans N. Ellemers, R. Spears et B. Doosje, Social Identity, Blackwell Publishers, p. 6-34, 1999.

Vermeulen, F. et Wang, T., « Imitation or distinction: Strategic responses to social reference groups ", communication EURAM, Munich, 4-7 mai 2005 et Academy of Management, Honolulu, 5-10 août 2005.

Zucker, L. G., "The Role of Institutionalization in Cultural Persistence ", American Sociological Review, Vol. 42, No. 5, p. 726-743, 1977. 